\title{
The importance of standardization in surgical ablation for atrial fibrillation
}

\author{
Niv Ad, MD
}

\author{
From the Department of Cardiac Surgery Research, Inova Heart and Vascular Institute, Falls Church, Va. \\ Disclosures: Dr Niv Ad is a member of the speaker's bureau for AtriCure; a consultant for Medtronic Inc; a proctor \\ and member of the speaker's bureau for LivaNova; a member of the advisory board of Nido Surgical; and \\ co-owner for Left Atrial Appendage Occlusion, LLC. \\ Received for publication Sept 23, 2015; accepted for publication Sept 25, 2015; available ahead of print Oct 24, \\ 2015. \\ Address for reprints: Niv Ad, MD, Department of Cardiac Surgery Research, Inova Heart and Vascular Institute, \\ 3300 Gallows Rd, Falls Church, VA 22042 (E-mail: niv.ad@inova.com or nivadmd@ hotmail.com). \\ J Thorac Cardiovasc Surg 2016;151:399-401 \\ $0022-5223 / \$ 36.00$ \\ Copyright (c) 2016 by The American Association for Thoracic Surgery \\ http://dx.doi.org/10.1016/j.jtcvs.2015.09.111
}

The cut-and-sew Cox maze procedure for atrial fibrillation (AF) was first performed in 1987; despite its success, however, it was considered too complex and thus never gained a great deal of popularity. ${ }^{1}$ The field of surgical ablation for AF was therefore developed around various ablation devices and operative schemes. Although significant improvements have been made in such approaches, the Cox maze III or IV procedure, performed according to the original design and with only those ablation devices that can produce consistent transmural lesions, remains the recognized gold standard for the surgical ablation of AF. In the vast majority of cases, inferior results reflect incomplete application of the lesions or technical errors. ${ }^{2-4}$

In contrast to the relatively limited use that initially characterized AF surgical ablation procedures, today these procedures are being much more widely performed by surgeons with a much wider variety of training, experience, skill, and education. As a result, standardization is lacking in the selection of ablation devices and appropriate lesion sets, as well as in the procedures used for follow-up and outcome reporting. This lack of standardization in education, experience, and training translates to a unique and challenging situation in surgical practice. Recognizing the need for more uniformity in reporting the results of surgical ablation, several large national and international professional societies and organizations, including the Heart Rhythm Society (HRS), the European Heart Rhythm Association, and the European Cardiac Arrhythmia Society, have established consensus-based recommendations for reporting outcomes of clinical trials of ablation techniques. ${ }^{5-9}$

Not only reported success rates but also definitions of success for surgical ablation vary widely. As a result, success rates reported in the literature range from $64 \%{ }^{10}$ to $98 \%$, a phenomenon that is unique in medicine. The reason for this variability is clear, but more work should be done to improve excellence in the field. Improved standardization in outcome prediction and improved consistency in reporting

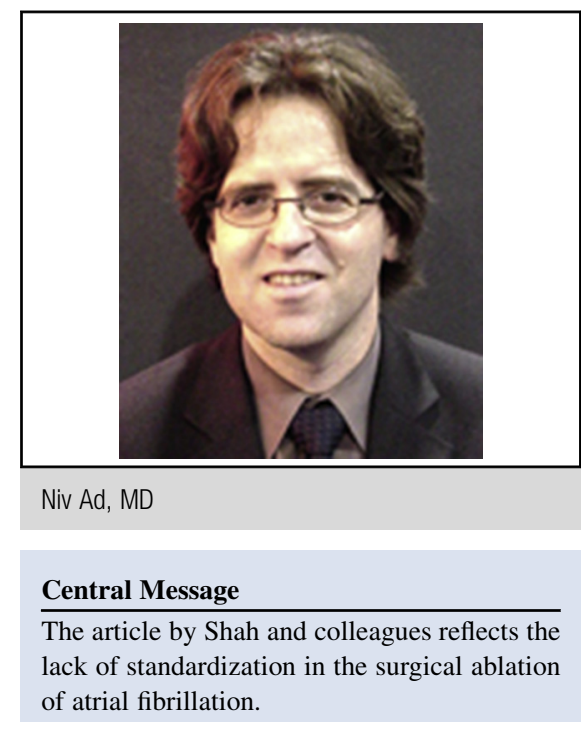

See Article page 391.

See Editorial page 298.

according to clearly established clinical indicators for success or failure are of highest priority. Failure in this regard complicates efforts to identify those patients who are most likely to benefit from surgical ablation procedures. We need to establish a reliable clinical process that includes appropriate training, as well as longitudinal indicators for outcome predictors for surgical ablation that can be used throughout clinical practice.

The study by Shah and colleagues ${ }^{12}$ in this issue of the Journal has explored the potential sex differences in outcomes of surgical ablation for AF. The research question posed by Shah and colleagues ${ }^{12}$ has been previously addressed in all aspects of treatments for AF, both medical and nonmedical. ${ }^{13,14}$ Recently, our group conducted a uniform analysis of patients undergoing a Cox maze III or IV procedure in which only those devices with proven consistency for transmurality were used (cryothermia either alone or in combination with bipolar radiofrequency; $\mathrm{N}=540$ ). ${ }^{13}$ We found that, despite the fact that female patients were at higher operative risk and came to surgery with higher rates of congestive heart failure and concomitant mitral valve surgeries than male patients, their outcomes were comparable to those of male patients. The rate at which female patients returned to sinus rhythm without being given antiarrhythmic 
drugs was the same, as were the 2-year survival rate and cardiac-related mortality. In addition, significant improvements in quality of life and in AF-related symptoms were documented for female patients. ${ }^{13}$

Shah and colleagues ${ }^{12}$ from Northwestern are to be congratulated for performing AF surgical ablation procedures in such high numbers, which exceed the national average. ${ }^{15}$ The article reflects their extensive experience with surgical ablation procedures that include many different ablation devices, some of which have been discontinued as a result of their inefficacy (laser and high-intensity focused ultrasound), as well as a wide variety of lesion sets. The latter were most often applied in the study on the basis of a single surgeon's discretion. Thus these decisions were often made not according to the disease process but rather on the basis of the concomitant procedure being performed. Shah and colleagues ${ }^{12}$ make it clear in the article's Discussion section that no sex differences in outcomes were found and that they believe the Cox maze procedure to be an inappropriate procedure in many cases.

The question that should be asked is whether Shah and colleagues ${ }^{12}$ have brought enough supporting evidence to their claim that the Cox maze procedure is overly aggressive. Their supporting reference for this claim is a recent New England Journal of Medicine study by Gillinov and colleagues ${ }^{16}$ that is characterized by some significant limitations, including inconsistent use of lesion sets and ablation technologies. The latter include unipolar radiofrequency, which is known to deliver inconsistent lesions. In addition, the study results of Gillinov and colleagues ${ }^{16}$ are not reported according to HRS guidelines, and most important, the study is greatly underpowered for a comparison of lesion sets, although Gillinov and colleagues ${ }^{16}$ correctly define the question of lesion set as a secondary end point. The New England Journal of Medicine article of Gillinov and colleagues ${ }^{16}$ is an important one, but in comparison with drug studies, multicenter, prospective, randomized cardiac surgery studies should be looked at very carefully, especially when such variability among centers and techniques is allowed within the study design.

Shah and colleagues ${ }^{12}$ report rhythm results at last followup, a practice that is not in accordance with HRS guidelines but is nevertheless often allowed and tolerated in the reporting of surgical ablation results. (Can you imagine a report on the patency rate of vein grafts in which the results of cardiac catheterization are compiled at 3 months in some patients and 10 years in others, and the patency rates are constructed from those recorded at last follow-up?) The HRS guidelines mandate that rhythm results should be presented at specific time points with clear definitions of success in the absence of class I or III antiarrhythmic drugs. ${ }^{9}$ Reporting freedom from $\mathrm{AF}$ at last follow-up does not provide reliable information about rhythm status, because there is likely to be vast variability in the time points at which patients had their last follow-up. In contrast, providing rhythm status at defined time points (eg, 6 months, 1 year, 2 years) provides clearer information to allow an adequate comparison between sexes. Shah and colleagues ${ }^{12}$ could perhaps maximize their follow-up data by also reporting on time to first $\mathrm{AF}$ recurrence. This approach, although clearly not able to adequately illustrate how patients move in and out of rhythm status, might indicate a pattern of earlier initial $\mathrm{AF}$ recurrence for one sex relative to the other, if such a pattern exists. Equally important is the unclear method with which patients who had catheter ablation for recurrence of atrial arrhythmia were defined in the study. The HRS guidelines clearly suggest that subsequent ablation procedures should be considered to have failed in such cases. ${ }^{9}$

Shah and colleagues ${ }^{12}$ astutely point out the very important challenge of working with cardiologists to standardize approaches during follow-up. Good communication and program development would address these issues. It is exceedingly difficult, however, to draw valid conclusions when using differing follow-up time points, lesion sets, and ablation devices, some of which are not consistent with reliable transmurality and were not selected according to an established protocol. Our group has faced the same challenges in a nonacademic environment (we are now following up more than 1000 patients operated on since 2005), but we have been able to establish the foundations for a protocol-based patient selection and follow-up process. ${ }^{17}$

It is also important to be aware of the fact that, in this study as in others, Shah and colleagues ${ }^{12}$ used different tools to define "success." The use of long-term monitoring systems is important, but how can electrocardiography be compared with long-term monitoring, which is significantly more sensitive in identifying atrial arrhythmias? Reported results should be clear about the methodology and devices used. The HRS guidelines suggest 24-hour Holter monitoring. ${ }^{9}$ Readers should be aware that in this case more is not necessarily better when it comes to monitoring, because data must be reviewed by an expert to rule out false-positive and false-negative results.

Shah and colleagues ${ }^{12}$ point out that, in light of the large numbers of surgical ablation procedures being performed today, it is important for these procedures to be done safely and effectively. In their Discussion section, Shah and colleagues $^{12}$ clearly state that the type of concomitant procedure performed, rather than the variables associated with $\mathrm{AF}$, was used to guide decisions about which lesion set to use. This would seem to suggest that it is perfectly fine to perform pulmonary vein isolation in cases where the left or the right atrium is not being opened (eg, coronary artery bypass graft or aortic valve replacement). Such an approach is not based on clinical data; to the contrary, recent studies found that the concomitant Cox maze procedure was not associated with increased risk for complications across different risk groups and procedures ${ }^{18,19}$ and that 
pulmonary vein isolation was associated with poor success rates only when performed concomitantly with aortic valve replacement, subjecting $50 \%$ of patients to postprocedural atrial arrhythmias. ${ }^{20}$ As surgeons, our goal should be to apply the correct lesion set in the appropriate cases, using only with those ablation tools that have been shown to produce consistent and reliable transmural lesions. The decision of which surgical ablation approach to use should be based only on the clinical variables associated with $\mathrm{AF}$ and other clinical justifications.

Shah and colleagues ${ }^{12}$ claim that one of the strengths of their study is the wide variety of lesion sets and devices used throughout the study period. I would be very careful before agreeing with this assertion. Clinical judgment and experience are extremely important, and although the Northwestern group has certainly mastered these, this study is a retrospective one in which a wide variety of approaches were allowed on the basis of surgeon discretion and a wide variety of methods were used during follow-up.

The results of the study show that sex is probably not associated with higher rates of failure of surgical ablation of AF. This conclusion fits well with what we know about the mechanism of $\mathrm{AF}$ and the predictors of surgical ablation failure. There is no reason to think that these differ by sex. It is important, however, to recognize that female patients are getting treated later in the course of their disease and as a result may have more risk factors that are predictive of failure. ${ }^{13}$ Numerous studies have determined that the most important variables associated with success of surgical ablation are age, size of the left atrium, type of AF, and, perhaps most important, duration of the arrhythmia.

It is essential to establish and adhere to a process for the different schemes of surgical ablation to improve patient selection and success rates. We must strive for excellence in surgical ablation by educating and training surgeons and cardiologists in the procedure. To that end, it is of utmost importance that we understand and standardize procedures for patient selection, ablation tools, and follow-up protocols for postprocedural treatment and reporting.

\section{References}

1. Cox JL, Canavan TE, Schuessler RB, Cain ME, Lindsay BD, Stone C, et al. The surgical treatment of atrial fibrillation. II. Intraoperative electrophysiologic mapping and description of the electrophysiologic basis of atrial flutter and atrial fibrillation. J Thorac Cardiovasc Surg. 1991;101:406-26.

2. Ad N, Henry L, Hunt S. The concomitant cryosurgical Cox-maze procedure using argon-based cryoprobes: 12-month results. J Cardiovasc Surg (Torino). 2011:52:593-9.

3. Damiano RJ Jr, Schwartz FH, Bailey MS, Maniar HS, Munfakh NA, Moon MR, et al. The Cox maze IV procedure: predictors of late recurrence. $J$ Thorac Cardiovasc Surg. 2011;141:113-21.

4. Cox JL, Ad N. New surgical and catheter-based modifications of the maze procedure. Semin Thorac Cardiovasc Surg. 2000;12:68-73.

5. Ad N, Cheng DC, Martin J, Berglin EE, Chang BC, Doukas G, et al. Surgical ablation for atrial fibrillation in cardiac surgery: a consensus statement of the International Society for Minimally Invasive Cardiothoracic Surgery (ISMICS) 2009. Innovations (Phila). 2010;5:74-83.
6. Calkins H, Brugada J, Packer DL, Cappato R, Chen SA, Crijns HJ, et al; Heart Rhythm Society; European Heart Rhythm Association; European Cardiac Arrhythmia Society; American College of Cardiology; American Heart Association; Society of Thoracic Surgeons. HRS/EHRA/ECAS expert consensus statement on catheter and surgical ablation of atrial fibrillation: recommendations for personnel, policy, procedures and follow-up. A report of the Heart Rhythm Society (HRS) task force on catheter and surgical ablation of atrial fibrillation developed in partnership with the European Heart Rhythm Association (EHRA) and the European Cardiac Arrhythmia Society (ECAS); in collaboration with the American College of Cardiology (ACC), American Heart Association (AHA), and the Society of Thoracic Surgeons (STS). Endorsed and approved by the governing bodies of the American College of Cardiology, the American Heart Association, the European Cardiac Arrhythmia Society, the European Heart Rhythm Association, the Society of Thoracic Surgeons, and the Heart Rhythm Society. Europace. 2007; 9:335-79.

7. Calkins H, Brugada J, Packer DL, Cappato R, Chen SA, Crijns HJ, et al; European Heart Rhythm Association (EHRA), European Cardiac Arrhythmia Society (ECAS), American College of Cardiology (ACC), American Heart Association (AHA), Society of Thoracic Surgeons (STS). HRS/EHRA/ECAS expert consensus statement on catheter and surgical ablation of atrial fibrillation: recommendations for personnel, policy, procedures and follow-up. A report of the Heart Rhythm Society (HRS) task force on catheter and surgical ablation of atrial fibrillation. Heart Rhythm. 2007;4:816-61. Erratum in: Heart Rhythm. 2009;6:148.

8. Shemin RJ, Cox JL, Gillinov AM, Blackstone EH, Bridges CR. Workforce on Evidence-Based Surgery of the Society of Thoracic Surgeons. Guidelines for reporting data and outcomes for the surgical treatment of atrial fibrillation. Ann Thorac Surg. 2007;83:1225-30.

9. Calkins H, Kuck KH, Cappato R, Brugada J, Camm AJ, Chen SA, et al. 2012 HRS/EHRA/ECAS expert consensus statement on catheter and surgical ablation of atrial fibrillation: recommendations for patient selection, procedural techniques, patient management and follow-up, definitions, endpoints, and research trial design. J Interv Card Electrophysiol. 2012; $33: 171-257$.

10. Gaynor SL, Diodato MD, Prasad SM, Ishii Y, Schuessler RB, Bailey MS, et al. A prospective, single-center clinical trial of a modified Cox maze procedure with bipolar radiofrequency ablation. J Thorac Cardiovasc Surg. 2004;128:535-42. Erratum in: J Thorac Cardiovasc Surg. 2006;131:772.

11. Saint LL, Bailey MS, Prasad S, Guthrie TJ, Bell J, Moon MR, et al. Cox-maze IV results for patients with lone atrial fibrillation versus concomitant mitral disease. Ann Thorac Surg. 2012;93:789-94; discussion 794-5.

12. Shah SV, Kruse J, Andrei AC, Li Z, Malaisrie SC, Knight BP, et al. Gender differences in outcomes following surgical ablation of atrial fibrillation. J Cardiovasc Thorac Surg. 2016;151:391-8.

13. Henry L, Hunt S, Holmes SD, Martin LM, Ad N. Are there gender differences in outcomes after the Cox-maze procedure for atrial fibrillation? Innovations (Phila). 2013;8:190-8.

14. Walters TE, Teh AW, Spence S, Morton JB, Kistler PM, Kalman JM. Absence of gender-based differences in the atrial and pulmonary vein substrate: A detailed electroanatomic mapping study. J Cardiovasc Electrophysiol. 2014; 25:1065-70.

15. Ad N, Suri RM, Gammie JS, Sheng S, O'Brien SM, Henry L. Surgical ablation of atrial fibrillation: trends and outcomes in North America. J Thorac Cardiovase Surg. 2012;144:1051-60.

16. Gillinov AM, Gelijns AC, Parides MK, DeRose JJ Jr, Moskowitz AJ, Voisine P et al; CTSN Investigators. Surgical ablation of atrial fibrillation during mitral-valve surgery. N Engl J Med. 2015;372:1399-409.

17. Ad N, Henry L, Hunt S, Stone L. The implementation of a comprehensive clinical protocol improves long-term success after surgical treatment of atrial fibrillation. J Thorac Cardiovasc Surg. 2010;139:1146-52.

18. Ad N, Henry LL, Holmes SD, Hunt SL. The impact of surgical ablation for atrial fibrillation in high-risk patients. Ann Thorac Surg. 2012;93:1897-903; discussion 1903-4.

19. Ad N, Holmes SD, Pritchard G, Shuman DJ. Association of operative risk with the outcome of concomitant Cox maze procedure: a comparison of results across risk groups. J Thorac Cardiovasc Surg. 2014;148:3027-33.

20. Henn MC, Lawrance CP, Sinn LA, Miller JR, Schuessler RB, Moon MR, et al. Effectiveness of surgical ablation in patients with atrial fibrillation and aortic valve disease. Ann Thorac Surg. 2015;100:1253-60. 